\title{
Transient cooling of two circular cylinders arranged in Tandem and bounded by an adiabatic wall
}

\author{
N. H. Saeid ${ }^{1}$, N. Hasan'1, Seri Rahayu Ya'akub¹, S. Shuib² \\ ${ }^{1}$ Faculty of Engineering, Universiti Teknologi Brunei, Jalan Tungku Link, Gadong BE 1410, Brunei Darussalam \\ Phone: +0673- 2461020 \\ ${ }^{2}$ Faculty of Mechanical Engineering, Universiti Teknologi Mara, Shah Alam, Selangor, Malaysia
}

\begin{abstract}
Parametric study is carried out on the transient cooling process of two circular cylinders in tandem arrangement for a specified period of time. Transient analysis of conjugate (conduction and convection) heat dissipation from two identical cylinders is considered with various parameters. The two cylinders of same size and properties are bounded by an adiabatic flat wall from below and the cooling air is flowing normal to their axis (cross flow). The following parameters are investigated in the present study: Reynolds number, cylinders thermal properties, separation distance between the two cylinders and the cooling time. The laminar flow is considered with Reynolds number values from 50 to 500 . The simulations are carried out for cooling the two cylinders made of carbon steels, plastics plexiglass and plywood. The local and average Nusselt number for both steady and transient cooling of the two cylinders are presented. The effects of the parameters are investigated and the results are presented to understand the process. It is found that increasing either the separation distance and/or the Reynolds number will increase the heat dissipation and reduce the cooling time. The results show that carbon steels cylinders need longer time of cooling compare with the plywood cylinders due to the difference in their thermal inertia.
\end{abstract}

ARTICLE HISTORY

Received: $13^{\text {th }}$ July 2020

Revised: $14^{\text {th }}$ Sept. 2021

Accepted: 09th Nov. 2021

\section{KEYWORDS}

Conjugate heat transfer;

transient analysis;

circular cylinders;

Tandem arrangement;

thermal inertia;

CFD simulations.

\section{INTRODUCTION}

Fluid dynamics over two cylinders arranged in tandem, in which one cylinder is placed downstream of the second cylinder, is important in many engineering applications and has a relatively complicated flow structure. The engineering application includes the flow over off shore structures, bridges, chimneys, cables, etc. Many studies have been carried out to determine the surface pressures and therefore the lift and drag forces for different flow conditions. Studies considering the cross flow over two cylinders without heat transfer can be found in references [1-4] among others.

A comprehensive review is presented by Sumner [1] for understanding the effect of placing two circular cylinders in cross flow on the flow field structure, aerodynamics loads, vortices and other parameters. Different arrangements of the cylinders were discussed, such as side by side, staggered or in tandem configurations. Harichandan and Roy [2] used numerical methods to study the flow behaviour past a single cylinder as well as tandem square and circular cylinders in vicinity of a wall with low Reynolds number ( $R e=100$ and 200). They studied the flow structure without heat transfer. Their analysis focused on the boundary layer growth and the locations of the cylinders' parameters. Their results are presented for different parameters to visualize vorticities and streamlines. Similarly, the wall effects on flow structure without heat transfer over two tandem cylinders is studied by Jiang and Lin [3] with low Reynolds number $(\mathrm{Re}=20$ 120). They implemented the Lattice Boltzmann numerical method to investigate the flow over two cylinders with fixed diameters ratio of 1.5 and spacing of 6 times the diameter of the small cylinder. They presented the numerical results for many cases, such as smaller cylinder in the upstream and the big cylinder upstream with different valves of Reynolds number, channel width and blockage ratio. Dehkordi et al [4] investigated numerically the unsteady flow over two circular cylinders arranged in tandem. Their results for different Reynolds number and spacing between cylinders show different flow structure and characteristics in laminar and turbulent flow regimes.

Forced and mixed (free and forced) convection cooling of cylinders has been considered in many studied in the past due to its importance in many industrial engineering practices. The applications include cooling/heating of cylinders in the heat treatments of metals, cooling of cylindrical foods in food processing and storage, cooling of electronic components, cooling towers and shell and tube heat exchanges among others. Many studies considered the mixed convection (forced and free) heat transfer across two or line of cylinder with various arrangements confined in horizontal and vertical ducts [5-9]. Sarkar et al. [5] studied the mixed convection mode for cooling two cylinders with square cross section in laminar flow with $\mathrm{Re}=100$. Two cases where considered in which the bouncy driven flow is in same direction with the external flow (aiding) and when it is in opposite direction (opposing). They reported that the cooling rate from the region of the cylinder in the downstream which is facing the flow is maximum and the flow become chaotic with severe thermal buoyancy conditions. Similarly, Lu et al [6] considered cooling or heating of two cylinders arranged in tandem with square cross section for numerical simulations using lattice Boltzmann method. The buoyancy effects also investigated in the study and the results are presented for Nusselt number and Richardson number for two values of 
Reynolds number in laminar regime. Similar studies carried out by Chatterjee et al. [7] and Chatterjee and Raja [8] where the unsteady mixed convection heat dissipation is considered from a row of cylinders with square cross section in crossflow. The flow fields along with thermal fields are presented for low Reynolds number and different separation distances and Richardson numbers. Harimi and Saghafian [10] simulated numerically the forced convection from isothermal circular cylinders arranged in tandem. Their results are presented for Nusselt number, lift and drag coefficients for different governing parameters. Laidoudi and Bouzit, [11] investigated the steady mixed convection heat dissipation from circular cylinders places in a horizontal channel in tandem arrangement. They presented the streamlines and isotherms and calculated the average Nusselt number under various conditions including Reynolds number, Richardson number and spacing between the cylinders. Recently Laidoudi and Makinde [12] extended the above study to analyse the aiding and opposing effects on the steady free and forced convection from circular cylinders placed in a vertical channel in tandem arrangement. They presented the streamlines and isotherms and calculated the average Nusselt number for many cases with various governing parameters.

Relatively few studies were found in the literature for the unsteady analysis of the convection heat transfer from two cylinders in tandem. Juncu [13-14] considered the unsteady numerical analysis for momentum transfer and convection heat transfer from circular cylinders arranged in tandem. The results are presented for the conduction dominated flow $(\operatorname{RePr}<1)$ which is different than the convection dominated flow $(\operatorname{RePr}>1)$. The results show that the drag of each cylinder in the case of tandem is less than the drag of the single cylinder case and the heat transfer is enhanced with high dimensionless temperatures of the cylinders. Mahir and Altaç [15] investigated the unsteady heat transfer from two circular cylinders in tandem arrangements with $\mathrm{Re}=100$ and 200 . The results are presented to show the effect of the separation distance of the cylinders on the drag and lift coefficients, Strouhal and Nusselt numbers. Salecedo et al [16] studied the unsteady mixed convection heat transfer from two semi-circular cylinders in tandem arrangement placed in a vertical channel. The results are presented for mean values and instantaneous variation of the velocities and temperatures. Their results show the effects of the buoyancy, separation distance, and wall confinement on flow structure, vortex dynamics and heat dissipation characteristics of both cylinders in laminar flow.

In all the above studies, the cylinders are arranged in the centre of the duct horizontally or vertically and the fluid flow around the whole surface. It is noticed that the unsteady convection heat transfer from two circular cylinders arranged in tandem and bounded by a wall is not received attention. The results of such analysis are very useful in many applications. For example, transient cooling/heating of cylinders placed on a surface in the heat treatments of metals, cooling of cylindrical foods placed on a surface in a refrigerator, cooling of cylindrical electronic components with different arrangement mounted on a board, etc. Similar investigations are carried out by Saeid and Busahmin [17] for a single cylinder and Martins et al [18] for two apples in tandem arrangement.

The aim of the present numerical simulation is to analyse the governing parameters of the transient conjugate cooling of two identical cylinders in tandem arrangement bounded by an adiabatic surface. Figure 1 shows the physical model with the coordinate geometry of the present problem.

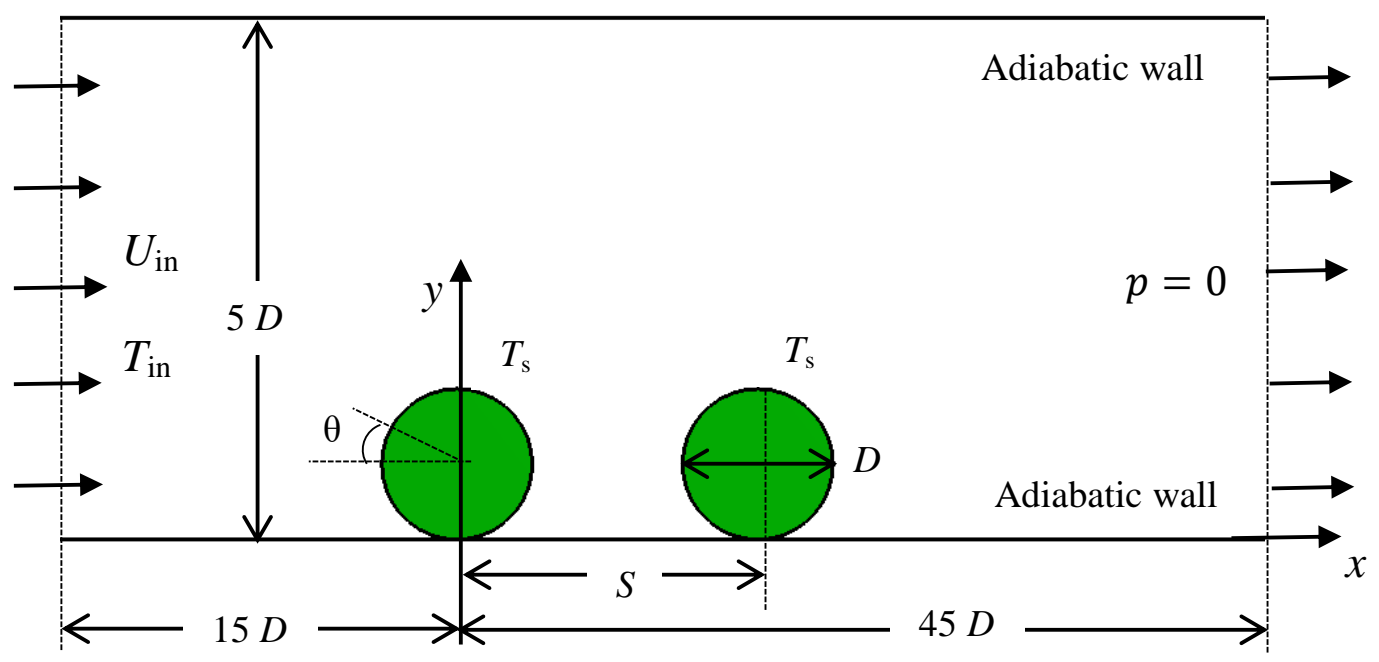

Figure 1. The physical model and coordinate system

\section{MATHEMATICAL MODEL}

The time dependent model is used for the conduction and convection heat transfer in the cooling of two cylinders arranged in tandem and placed on an adiabatic surface in a duct shown in Figure 1. It is assumed that hot cylinders are placed in a cold air stream in cross flow. The heat is dissipated from the interior of the cylinders to the surface by conduction mode and from its surfaces to the air by convection. In the present study air is used as the cooling fluid, which is assumed with constant properties including the density, and the buoyancy effect is neglected. The flow across the 
cylinders is assumed to be two dimensional neglecting the effect of the cylinder length which is valid assumption for long cylinders such as cooling of steel bars in the heat treatment process.

The size of the duct is extended (as shown in Figure 1) to minimize the effects of the duct walls and inlet and exit boundary conditions on the cooling process. The distances upstream and downstream are selected to be $15 D$ and $45 D$, respectively and the distance between the lower and upper walls is $5 D$ as shown in Figure 1. Computational tests were performed to find that higher values give almost similar results for the flow region around the cylinders. Under the present geometry and assumptions, the mathematical model for the transient conjugate heat transfer can be presented as:

$$
\nabla \cdot \vec{V}=0
$$

$$
\begin{gathered}
\frac{\partial(\rho u)}{\partial t}+\nabla \cdot(\rho u \vec{V})=-\frac{\partial p}{\partial x}+\nabla \cdot(\mu \nabla u) \\
\frac{\partial(\rho v)}{\partial t}+\nabla \cdot(\rho v \vec{V})=-\frac{\partial p}{\partial y}+\nabla \cdot(\mu \nabla v) \\
\rho c_{p} \frac{\partial T}{\partial t}+\rho c_{p} \nabla \cdot(\vec{V} T)=\nabla \cdot(k \nabla T)
\end{gathered}
$$

where the velocity vector $\vec{V}$ has the components $u$ and $v$ in the $x$ and $y$ directions, respectively. The heat conduction equation for the heat transfer inside the cylinders is:

$$
\left(\rho c_{p}\right)_{s} \frac{\partial T_{s}}{\partial t}=\nabla \cdot\left(k_{s} \nabla T_{s}\right)
$$

where subscript $s$ represents solid cylinder. The air velocity is set to be zeros on all solid surfaces (no slip condition). The boundary duct walls are considered adiabatic (thermally insulated). On the cylinder surfaces the coupled thermal boundary condition is implemented as follows:

$$
\begin{gathered}
T_{s}=T \\
k_{s}\left(n \cdot \nabla T_{s}\right)=k(n \cdot \nabla T)
\end{gathered}
$$

where $n$ is the normal vector direction to the solid-fluid interface. The inlet boundary condition is imposed as uniform velocity and temperature ( $U_{\text {in }}$ and $T_{\text {in }}$ ) of the air. At the outlet, atmospheric pressure is used for the momentum equations and zero conduction heat flux normal to the exit boundary is imposed for energy equation. The cylinders are assumed to be identical in size, materials and initial temperatures.

Three different materials are examined in the present study. The properties of the working fluid and cylinders materials are listed in Table 1.

Table 1. Physical properties of the air and the solid cylinders [19]

\begin{tabular}{ccccc}
\hline Property & Air & Carbon steels & $\begin{array}{c}\text { Plastics } \\
\text { plexiglass }\end{array}$ & Plywood \\
\hline density $\rho\left(\mathrm{kg} / \mathrm{m}^{3}\right)$ & 1.225 & 7854 & 1190 & 545 \\
specific heat $c_{p}(\mathrm{~J} / \mathrm{kgK})$ & 1006 & 434 & 1465 & 1210 \\
thermal conductivity $k(\mathrm{~W} / \mathrm{mK})$ & 0.0242 & 60.5 & 0.19 & 0.12 \\
Viscosity $\mu(\mathrm{kg} / \mathrm{ms})$ & $1.789 \times 10^{-5}$ & - & - & - \\
\hline
\end{tabular}

\section{NUMERICAL SCHEME}

The continuity, momentum and energy equations with the specified boundary conditions, defined above, are solved using numerical techniques build in FLUENT software [20]. This software using the finite volume method where the solution domain is divided into finite number of cells (or control volumes) and integrates the governing equations over the control volumes. Therefore, the duct shown in Figure 1 is meshed by discretising it into quadrilateral cells. Unstructured mesh is generated using ANSYS software [21] so that the faces of the cells are aligned with the interface between the cylinder wall and the air surrounding it. This is important to get accurate implementation of the boundary condition defined in Eq. 6(b). 
Figure 2 shows the mesh geometry (for the case of $S=3 D$ ) in the region near the cylinders where high gradients of the velocities, pressure and temperatures are expected. The moderated size mesh is shown in Figure 2 and the gird independency test will be discussed in the next section.

The implicit scheme is implemented for discretization of time with first order and time step of 30 seconds. The least squares gradient evaluation is used for the spatial discretization. In this method, the values of the velocities, pressure and temperatures are approximated by linear variation between the cells centres. In the discretization of the last term in Eqs. (2) to (5), which is called the diffusion terms, the central differencing scheme is used. The second order upwind scheme is recommended for more accurate simulations and used in the present study for the convection-diffusion terms discretization of the momentum and energy equations. For velocity-pressure coupling the pressure-based coupled algorithm is found to be more accurate than others in a relatively short computation time. The iterative solution method is used to solve the discretised linear equations and the values of the explicit relaxation factors are 0.5 for both momentum and pressure. The flow Courant number of 200 is used while the under-relaxation factor for energy is kept unity.

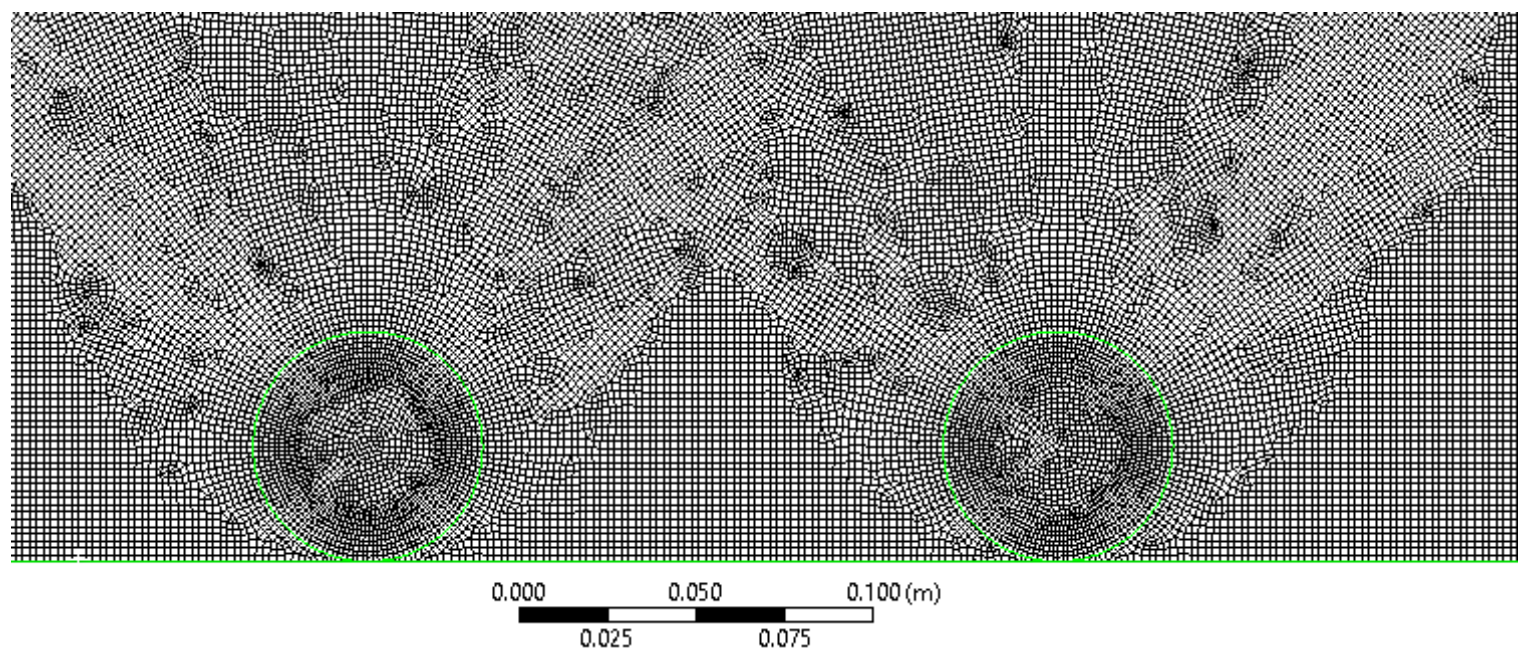

Figure 2. Mesh geometry near the cylinders for $S=3 D$ (Total number of cells $=321401)$

The iterative solution starts with the initial guess for all the dependent variables and continue with the iterative calculations until the convergence criterion is met. The maximum absolute residual was $10^{-9}$ in the energy equation and the absolute residuals of the velocity components were lower than $10^{-6}$ in the final solution. The overall heat and mass balances are used for verification of the accuracy of the present numerical method. The differences between the inlet and outlet heat fluxes and momentum fluxes in the converged solution are calculated and found within $\pm 10^{-3} \%$ in all simulation cases in the present study. Similar solution strategy is used successfully in the previous studies in the references [22-24].

\section{RESULTS AND DISCUSSION}

The mathematical model defined above is solved numerically using FLUENT software [20]. The solution generates the velocity components, pressure and temperature in the centre of each cell in the mesh. The convection heat transfer process is evaluated using the dimensionless parameter, Nusselt number. In the present study, the Nusselt number is defined based on the cylinder diameter $D$ as the length scale:

$$
N u_{\theta}=\frac{-(\partial T / \partial n)_{\theta} D}{\left(T_{s \theta}-T_{i n}\right)}
$$

where $(\partial T / \partial n)_{\theta}$ is the temperature derivative normal to the solid-fluid interface and $T_{s \theta}$ is the surface temperature of the cylinder at a location $\theta$ with the horizontal as shown in Figure 1. The average Nusselt number at the cylinder wall is calculated by the area weighted average over the whole cylinder surface as follows:

$$
\overline{N u}=\frac{1}{\pi D} \int_{0}^{\pi D} N u_{\theta} d s
$$

where $d s$ is the circumferential element size on the cylinder surface. The results will be presented for various values of the inlet velocity of the air which can be grouped with the properties of the air as Reynolds number. The cylinder diameter $D$ is used as the length scale for Reynolds number calculation as: $\left(\operatorname{Re}=\rho U_{i n} D / \mu\right)$.

The gird independency tests are carried out to study the effect of the mesh size on the results. To validate and verify the accuracy of the present solution strategy, three sizes were used to generate the results which are compared with the 
results obtained from other studies for the steady flow across a single isothermal cylinder. In the steady flow case, the surface temperature is maintained constant as $T_{s}=300 \mathrm{~K}$ in the boundary condition (6). This surface temperature is used as $T_{s \theta}$ in the calculation of the Nusselt number in eq. (7).

The local and average Nusselt number around the cylinder with referenced values of the same flow across a single circular cylinder are calculated. Figure 3 shows the local Nusselt number variation around the isothermal cylinders compared with the results presented by Saeid and Busahmin [17] for a similar flow over one cylinder. The results presented in Figure 3 are generated using the moderated size mesh. Obviously, the present results for the upstream cylinder are very close to the results of the single cylinder for low Reynolds number and large separation distance $S$ as shown in Figure 3(a) for $\operatorname{Re}=50$. At high Reynolds number $(\operatorname{Re}=500)$ the present results are also in agreement with those of the single cylinder except the region $120^{\circ} \leq \theta \leq 240^{\circ}$ where the downstream cylinder location is affecting the flow, and therefore, the cooling process. It is obvious that, the flow around the downstream cylinder is not comparable with the flow across a single cylinder. The variation of the Nusselt number around the downstream cylinder is presented in Figure 3(b) for comparison and not for validation purpose. The maximum local Nusselt number is found in the region $40^{\circ} \leq \theta \leq 50^{\circ}$ for the cylinder facing the air first (upstream). While maximum value of the local Nusselt number occurred at higher angles $\left(\theta>50^{\circ}\right)$ and its location depends on the separation distance and the flow velocity for the downstream cylinder as shown in Figure 3(b). For all cases and both cylinders, the local Nusselt number shows a minimum value at $\theta=270^{\circ}$ which is at the adiabatic boundary wall as shown in Figure 1.

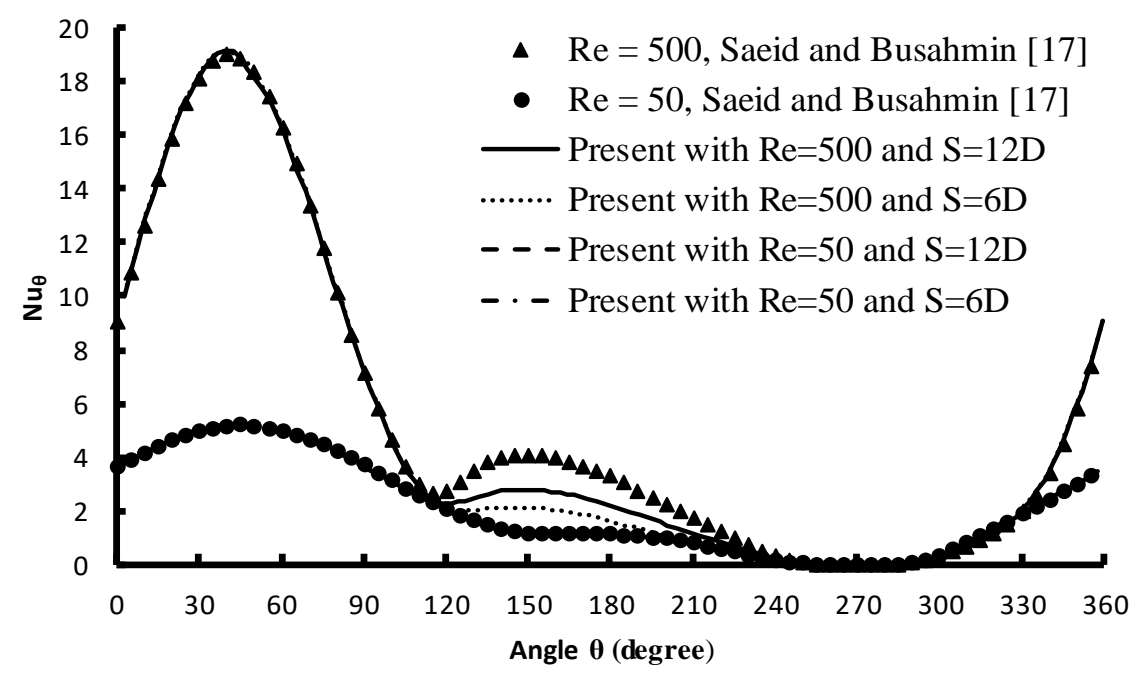

(a) Upstream cylinder

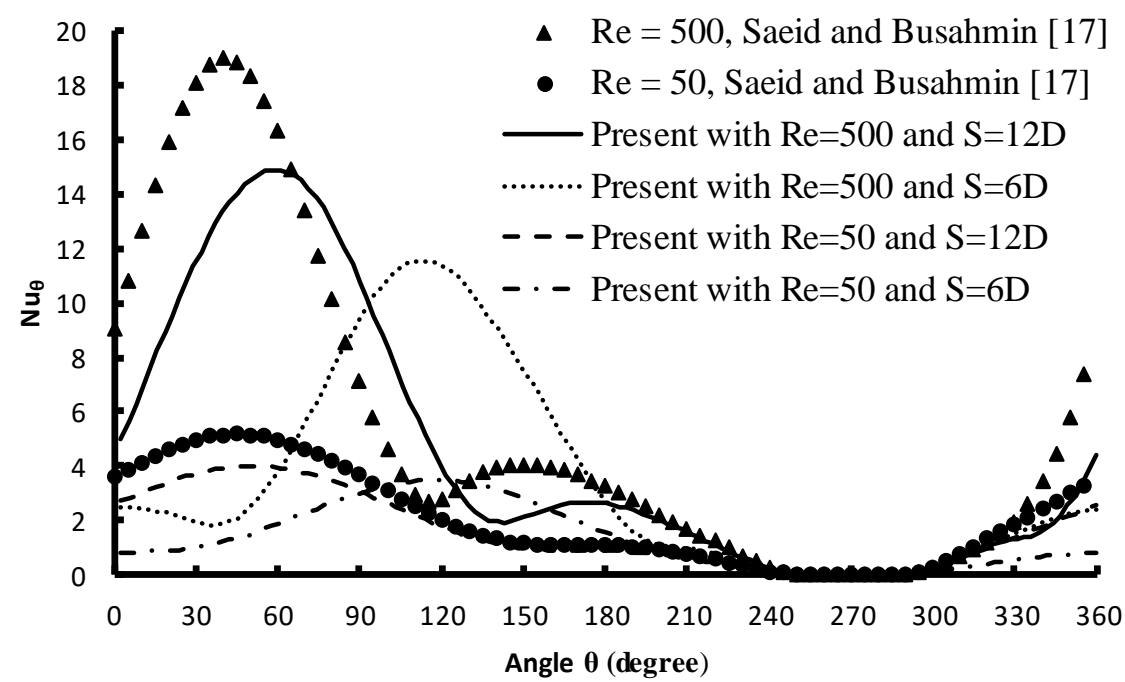

(b) Downstream cylinder

Figure 3. Variation of $N u_{\theta}$ for steady flow across isothermal cylinder bounded by an adiabatic wall

The average Nusselt number is calculated by integration the curves presented in Figure 3 using Eq. (8) and the results of the area weighted average values are listed in Table 2. As observed from the local Nusselt number variation around the upstream cylinder, it is approaching the respective values of the flow around a single cylinder at low Reynolds number and large separation distances. The average Nusselt number for the flow with $\mathrm{Re}=50$ is approaching the respective value for the flow around a single cylinder for all mesh sizes and both $S=6 D$ and $S=12 D$ as shown in Table 2. As Re increases 
the differences between the average Nusselt number values between the present study and the results from single cylinder increases. Obviously, this is due to the location of the downstream cylinder and its effects on the flow behind (wake region) the upstream cylinder with increasing the inlet velocity.

The results presented in Table 2 show that the average Nusselt number values calculated from the downstream cylinder are less than those of the upstream cylinder for the present identical cylinders in tandem arrangement. The heat lost by convection from the downstream cylinder is less than that of the upstream cylinder because the air flow around the downstream is disturbed and preheated by the upstream cylinder. The differences between the values of the Nusselt number of the upstream and downstream cylinders can be minimized by increasing the separation distance $S$ as shown in Table 2.

Values of the Nusselt number calculated using different mesh sizes show small differences as listed in Table 2. The maximum difference in the values obtained using the coarse mesh and moderated mesh is $1.53 \%$ and it is $0.56 \%$ between the results of moderated mesh and fine mesh. Therefore, the results presented in Figure 3(a) and Table 2 validate the present solution method and the size of the mesh used. Therefore, the moderate size mesh is considered for results generation of the following parametric study.

Table 2. Average Nusselt number for steady flow around an isothermal cylinder bounded with adiabatic wall

\begin{tabular}{|c|c|c|c|c|c|c|c|}
\hline \multirow{3}{*}{$\operatorname{Re}$} & \multirow{3}{*}{$\begin{array}{c}\text { Single } \\
\text { cylinder } \\
{[17]}\end{array}$} & \multicolumn{6}{|c|}{ Present results of two cylinders in tandem arrangement with $S=6 D$} \\
\hline & & \multicolumn{2}{|c|}{$\begin{array}{l}\text { coarse mesh } \\
(143971 \text { cells })\end{array}$} & \multicolumn{2}{|c|}{$\begin{array}{l}\text { moderated mesh } \\
(321401 \text { cells })\end{array}$} & \multicolumn{2}{|c|}{$\begin{array}{c}\text { fine mesh } \\
(571283 \text { cells })\end{array}$} \\
\hline & & $\begin{array}{l}\text { Upstream } \\
\text { cylinder }\end{array}$ & $\begin{array}{c}\text { Downstream } \\
\text { cylinder }\end{array}$ & $\begin{array}{l}\text { Upstream } \\
\text { cylinder }\end{array}$ & $\begin{array}{c}\text { Downstream } \\
\text { cylinder }\end{array}$ & $\begin{array}{l}\text { Upstream } \\
\text { cylinder }\end{array}$ & $\begin{array}{c}\text { Downstream } \\
\text { cylinder }\end{array}$ \\
\hline 50 & 2.053 & 2.034 & 1.335 & 2.031 & 1.334 & 2.030 & 1.333 \\
\hline 100 & 2.736 & 2.634 & 1.839 & 2.629 & 1.837 & 2.626 & 1.834 \\
\hline 200 & 3.669 & 3.459 & 2.512 & 3.444 & 2.507 & 3.437 & 2.502 \\
\hline 300 & 4.358 & 4.085 & 2.951 & 4.057 & 2.941 & 4.044 & 2.933 \\
\hline 400 & 4.930 & 4.615 & 3.276 & 4.567 & 3.261 & 4.547 & 3.251 \\
\hline \multirow[t]{2}{*}{500} & 5.435 & 5.091 & 3.525 & 5.013 & 3.502 & 4.986 & 3.488 \\
\hline & & \multicolumn{6}{|c|}{$S=12 D$} \\
\hline 50 & 2.053 & 2.052 & 1.608 & 2.050 & 1.607 & 2.049 & 1.606 \\
\hline 100 & 2.736 & 2.739 & 2.003 & 2.733 & 2.001 & 2.730 & 2.000 \\
\hline 200 & 3.669 & 3.566 & 2.960 & 3.554 & 2.951 & 3.546 & 2.946 \\
\hline 300 & 4.358 & 4.192 & 3.606 & 4.169 & 3.592 & 4.155 & 3.585 \\
\hline 400 & 4.930 & 4.726 & 4.091 & 4.688 & 4.073 & 4.666 & 4.062 \\
\hline 500 & 5.435 & 5.211 & 4.501 & 5.147 & 4.476 & 5.118 & 4.461 \\
\hline
\end{tabular}

The constant stream function lines (streamlines) and constant temperature lines (isotherms) are shown in Figure 4 for steady flow across two isothermal cylinders. In these plots, the surface temperature is $300 \mathrm{~K}$ and inlet temperature is 275 $\mathrm{K}$ with low Reynolds number $(\mathrm{Re}=50)$ and high Reynolds number $(\mathrm{Re}=500)$. The effect of the distance between the two cylinders arranged in tandem can be seen from the contour plots presented with separation distances of $S=6 D$ and $S$ $=12 \mathrm{D}$.

As discussed above, the maximum local Nusselt number is found in the region $40^{\circ} \leq \theta \leq 50^{\circ}$ (near the front stagnation point) for the upstream cylinder as shown in Figure 4 for all the stream lines plots. The isotherms are cluster in this region $\left(40^{\circ} \leq \theta \leq 50^{\circ}\right)$ for the upstream cylinder for all cases shown in Figure 4 .

In general, the clustered isotherms create high temperature gradient, and therefore, high heat flux and high values of Nusselt number. In the flow around the downstream cylinder, the region of the clustered isotherms is shifted and rotated toward increasing the angle $\theta$ especially for the cases with low separation distances $(S=6 D)$. When the separation distance increased to $S=12 \mathrm{D}$, the upstream cylinder effect is minimized. The air flow field close to the downstream cylinder can approach that around the upstream one especially for the flows with low Reynolds number as shown in Figure 4 for the case $\operatorname{Re}=50$ and $S=12 \mathrm{D}$. Similar flow patterns are expected for other case with different Reynolds number and separation distances.

The results are generated to show the transient effects on the conjugate heat transfer from the two cylinders. The heat dissipation is happened by conduction mode inside the cylinder material and then by convection mode from its surface. This is known as conjugate heat transfer. In the transient analysis, the boundary condition defined in Eq. (6) is imposed on the cylinders' surfaces instead of the constant temperature. Therefore, the cylinders' surface temperatures are not constant and depending on the cooling time and conditions. In the present investigations, same initial conditions are used 
for all the cases considered, which are: at time $=0$ the air is stagnant with atmospheric pressure around the cylinders and temperature of the air and both cylinders is $300 \mathrm{~K}$. In this time, the air starts to flow with inlet temperature of $275 \mathrm{~K}$ and a specific velocity, which is calculated form the Reynolds number for the respective case.
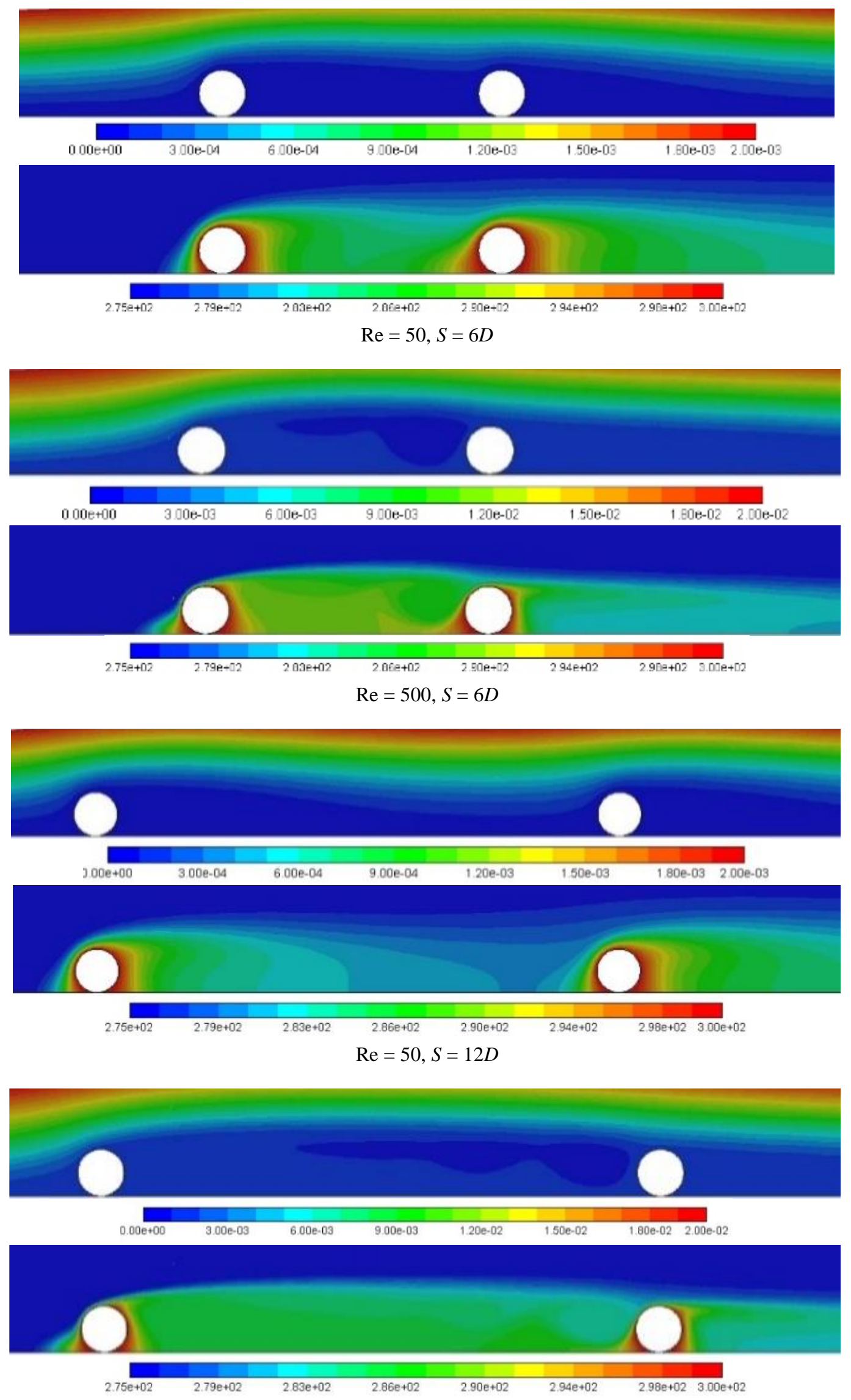

Figure 4. Streamlines, $\mathrm{kg} / \mathrm{s}$ (top) and isotherms, K (bottom) for steady flow across two isothermal cylinders 
The results are generated using the materials properties of the three cylinders listed in Table 1 . The cooling process is monitored with time for a period of 2 hours as depicted in Figure 5 for the two cylinders in tandem with separation distance of $S=3 D$ and low and high Reynolds number.

The isotherms plots presented in Figure 5 show the cooling stages of the cylinders where the cylinder surface and inside temperature decreasing with time.

It can be seen from Figure 5 that cooling period to a specific temperature will takes relatively longer time for the cylinders made of carbon steels. The cylinders made of plywood are the fastest to be cooled and the plastics plexiglass cylinders are taking a moderated cooling time. In all case shown in Figure 5, the maximum Nusselt number is found in the region $40^{\circ} \leq \theta \leq 50^{\circ}$ for the upstream (left side) cylinder (near the front stagnation point). Therefore, it is cooled faster than other regions and the isotherms shows minimum values at this region as shown in Figure 5. While the isotherms depicted in Figure 5 for the downstream (right side) cylinder indicate the region which is cooled faster are located at higher angles than the respective cases of the upstream cylinder. The isotherms presented in Figure 5 show the regions near the adiabatic boundary at $\theta=270^{\circ}$ show maximum temperatures for all cases. Therefore, it is expected to take relatively longer time to cool this region to the required thermal state.
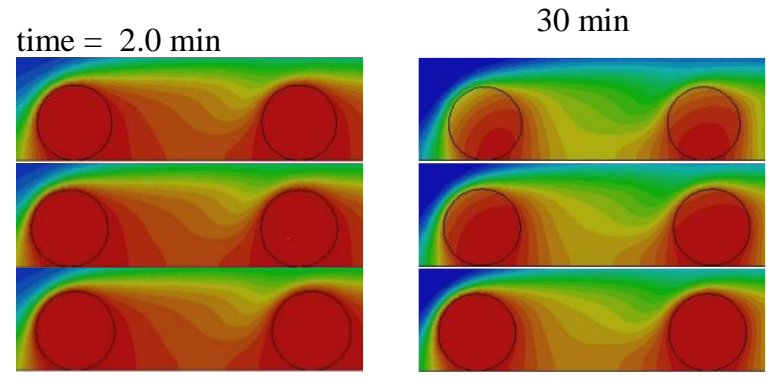

$60 \mathrm{~min}$

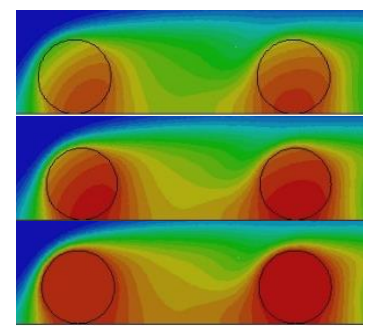

(a) $\mathrm{Re}=100$
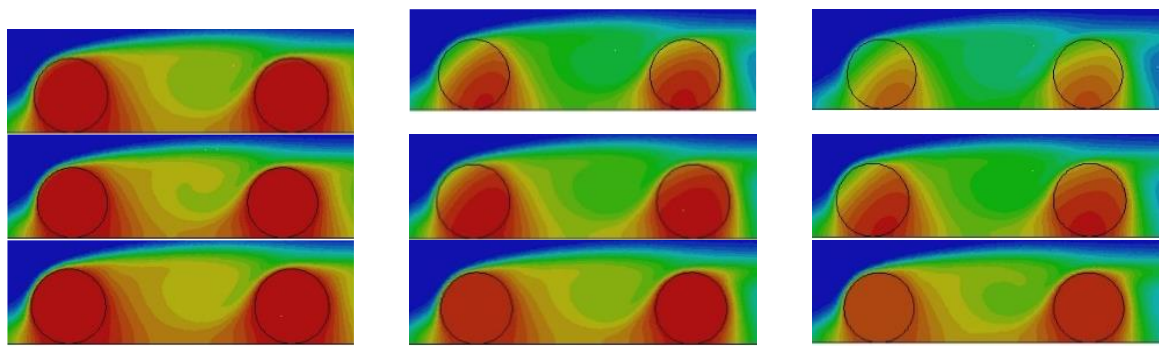

(b) $\operatorname{Re}=500$
$120 \mathrm{~min}$
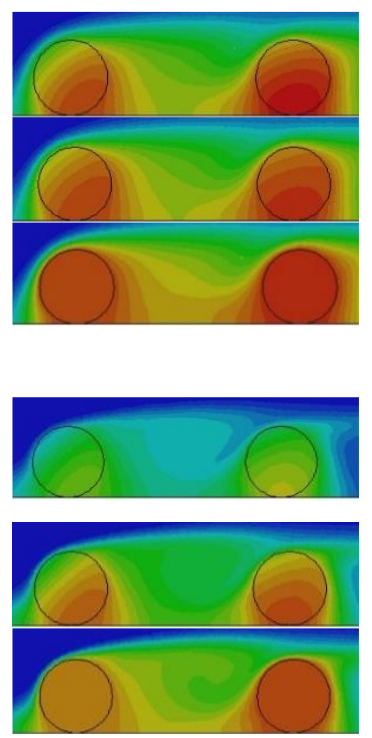

$98 e+023.00 e+02$

Figure 5. Isotherms $(\mathrm{K})$ near the two cylinders in tandem with $S=3 D$ (Top, Plywood; middle, Plastics Plexiglass; bottom, Carbon steels)

The direction of the heat dissipation is always from the region of maximum temperature to the region of minimum temperature inside the cylinders. Thus, the heat transfer direction inside the cylinders can be predicted by inspecting the isotherms presented in Figure 5 and 6. For the upstream cylinder, the heat dissipation direction is from the region at $\theta=$ $270^{\circ}$ to the region at $40^{\circ} \leq \theta \leq 50^{\circ}$. For the downstream cylinder, the heat dissipation direction is also from the region at $\theta=270^{\circ}$ to the region at $50^{\circ} \leq \theta \leq 90^{\circ}$. Comparing the results presented in Figure 5 (a) and Figure 5(b) reveal the importance of increasing the cooling air velocity (or Reynolds number) for faster cooling of the cylinders.

To investigate the effect of the distance between the two cylinders on the transient cooling, the results are generated and presented in Figure 6 for various separation distances after 2 hours of cooling. Similar observations discussed above for the cases with $S=3 D$ are noted with various separation distances. However, increasing the separation distance leads to faster cooling of the downstream (right side) cylinder for all cases as shown in Figure 6.

To compare the time required to cool the cylinders to a specific average temperature, the area weighted average temperature at the cylinder surface is introduced and calculated as:

$$
T_{a v}=\frac{1}{\pi D} \int_{0}^{\pi D} T_{\theta} d s
$$

The area weighted average temperature at the cylinders' surfaces are calculated and plotted against the cooling time for different parameters as shown in Figure 7 for upstream and downstream cylinders. Figure 7 shows that the area weighted average surface temperature of the cylinders are decreasing sharply for the cases of the plywood cylinders compare with others for all Reynolds numbers cases. This is due to the properties of the materials listed in Table 1. These properties can be combined in a single parameter called thermal inertia, which is defined (Wang et al. [25]) as: 


$$
I=\sqrt{k \rho c_{p}}
$$

The values of the thermal inertia of the materials listed in Table 1 can be calculated as: $I_{\text {wood }}=281.3 \mathrm{Jm}^{-2} \mathrm{~K}^{-1} \mathrm{~s}^{-1 / 2}$, $I_{\text {plastic }}=575.5 \mathrm{Jm}^{-2} \mathrm{~K}^{-1} \mathrm{~s}^{-1 / 2}$ and $I_{\text {steel }}=14360.4 \mathrm{Jm}^{-2} \mathrm{~K}^{-1} \mathrm{~s}^{-1 / 2}$. Therefore, it can be concluded that materials with low thermal inertia can be cooled faster than those with high thermal inertia.
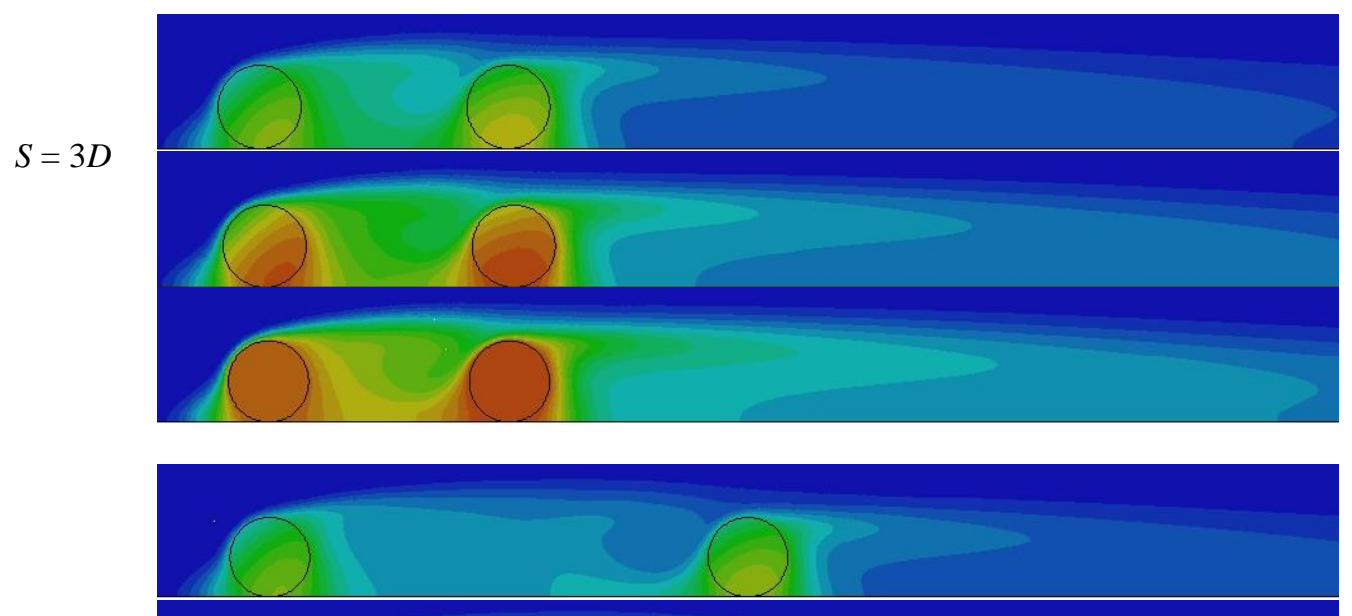

$S=6 D$
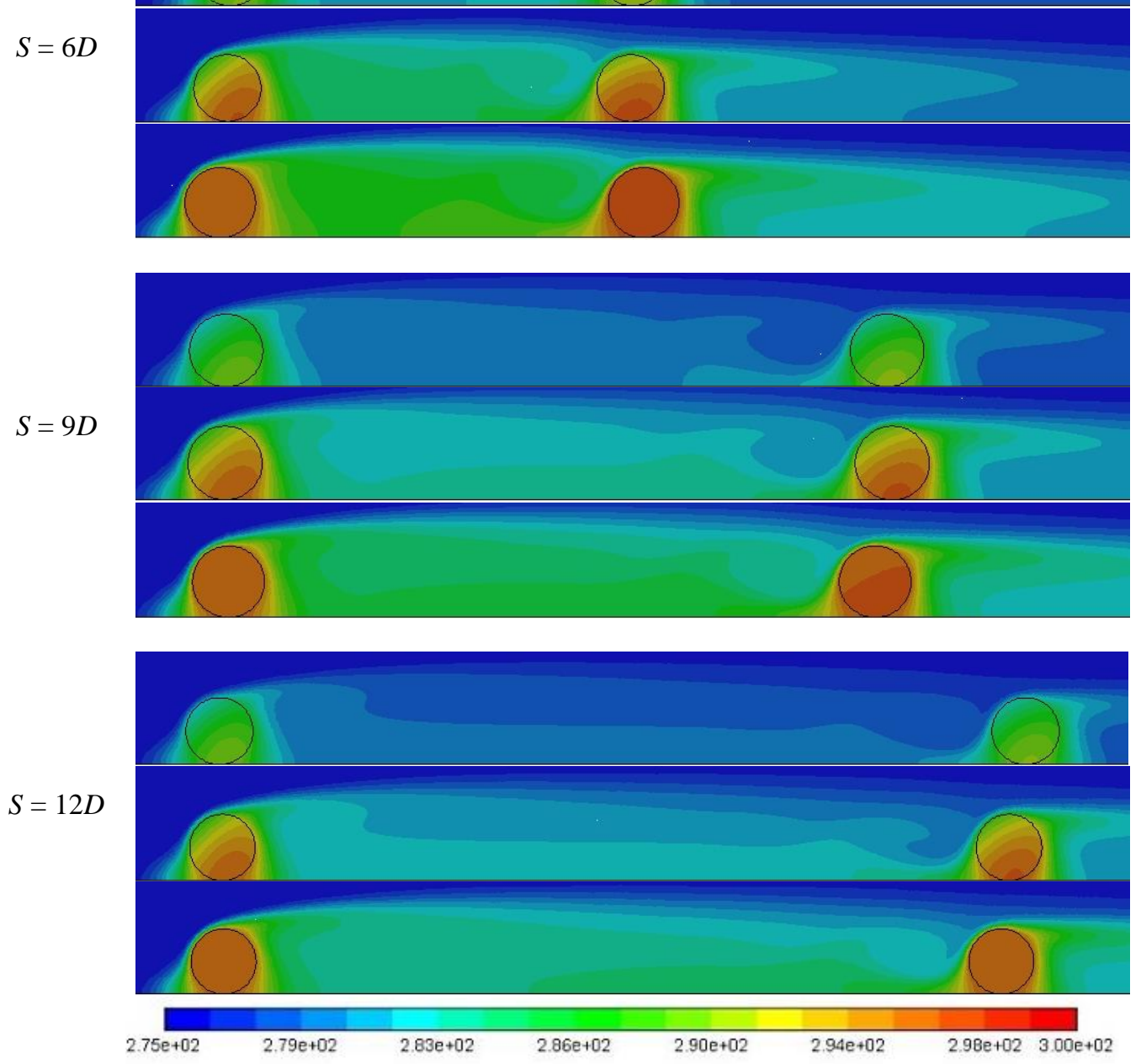

Figure 6. Isotherms (K) after 120 min of cooling by air flow with Re = 300 (Top, Plywood; Middle, Plastics Plexiglass; Bottom, Carbon steels)

The results presented in Figure 7 show that the average temperature of the cylinder surface can also be reduced by increasing the separation distance between the two cylinders especially for the downstream cylinder. For example, the average surface temperature of the downstream cylinder is reduced from $300 \mathrm{~K}$ to $289.1 \mathrm{~K}(3.65 \%)$ after 2 hours of 
cooling of plywood cylinders with $\mathrm{Re}=300$ and separation distance of $3 D$. Increasing the separation distance to $12 D$ the same cylinder under same conditions will be cooled from average surface temperature of $300 \mathrm{~K}$ to $286.3 \mathrm{~K}(4.55 \%)$.

Comparing the cooling of different materials, Figure 7 shows for the case of $\operatorname{Re}=300$ and $S=12 D$, the percentage decrease in the average surface temperature of the downstream cylinder is $4.55 \%$ in the plywood cylinder, $2.44 \%$ in the plastic cylinder and it is only $1.32 \%$ in the carbon steel cylinder. This is due to the difference in the thermal inertia of the materials.

The transient variation of the Nusselt number is presented in Figure 8 for the upstream and downstream cylinders. At the beginning of the cooling process the air around the cylinders is at same temperature with the cylinders and therefore the heat transfer rates and Nusselt number are zeros. As the cold air reaches the cylinders, the convection heat transfer starts to cool the cylinder and therefore the average value of $\mathrm{Nu}$ increased. If the process is continued, the heat transfer and the Nusselt number increased to reach maximum value after a period, which depends on the cylinder materials and flow conditions as shown in Figure 8.

For the upstream cylinder the transient variation of average Nusselt number is almost same for all separation distances and small variation for various materials. Similar observation is highlighted for the steady flow cases as presented in Figure 3 for local Nusselt number.

The transient values of the average Nusselt number of the downstream cylinder is always less than the respective values of the upstream cylinder. The variation of the average Nusselt number of the downstream cylinder show an increase with increasing the separation distance as presented in Figure 8(b). This Figure show also that the average Nu of the plywood cylinder is maximum and for carbon steel in minimum for all time steps during the cooling process. Again, the reason behind this variation is the cylinders with high thermal inertia will lose heat slower than those with low thermal inertia.

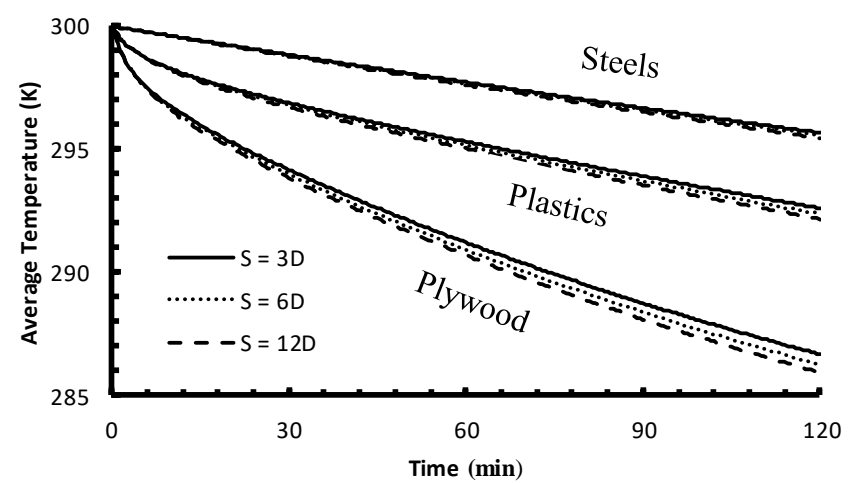

(a) Upstream cylinder

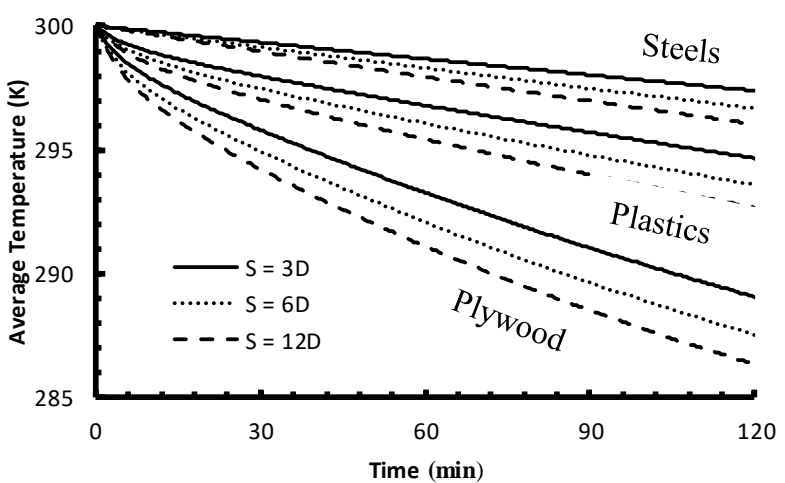

(b) Downstream cylinder

Figure 7. Transient variation of the average temperature at the cylinder surface with $\mathrm{Re}=300$

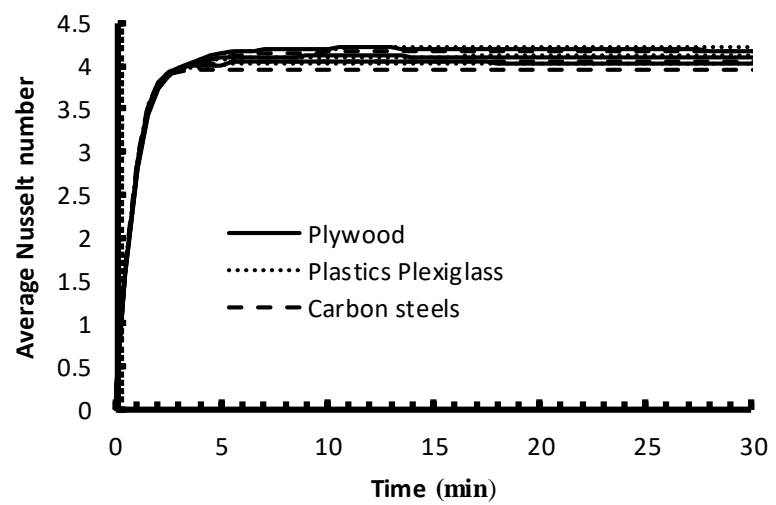

(a) Upstream cylinder

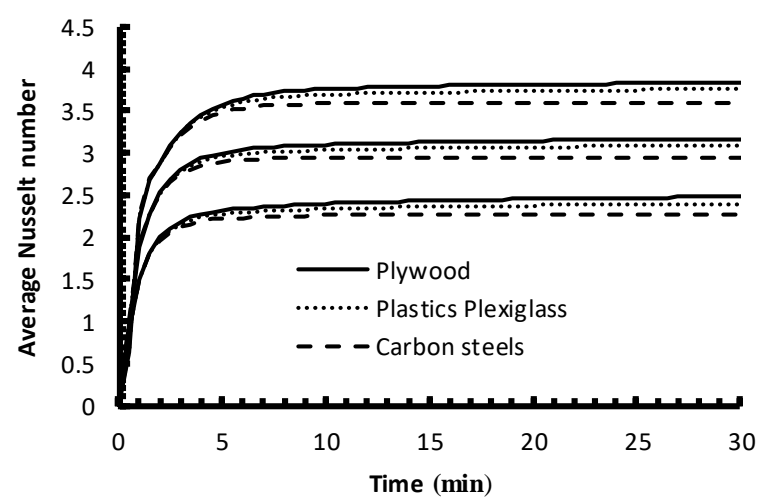

(b) Downstream cylinder

Figure 8. Transient variation of the average Nusselt number with $\operatorname{Re}=300$ (upper $S=12 D$; middle $S=6 D$; lower $S=$ $3 D)$

\section{CONCLUSIONS}

Transient numerical analysis is carried out for the conjugate heat dissipation from two identical cylinders arranged in tandem. The two cylinders are bounded by an adiabatic surface and the cooling fluid (air) is flowing across the cylinders. The parameters considered in the present study are: Reynolds number in laminar regime, cylinders thermal properties, spacing between the two cylinders and the cooling time period. Three materials are selected according to their thermal inertia. They are carbon steels with high thermal inertia, plastics plexiglass with moderated thermal inertia and plywood 
with low thermal inertia. For validation and verification purposes the results for the steady heat dissipation from two isothermal cylinders are presented with acceptable accuracy.

The transient cooling of the non-isothermal cylinders in tandem arrangement is monitored with time for 2 hours with various parameters. At the beginning of the cooling process the rate of heat transfer and the Nusselt number are zeros. By increasing the cooling time, the heat transfer increasing and the Nusselt number increased to reach maximum value after a period of time, which depends on the cylinder materials and flow conditions. The results show that the maximum value of the local Nusselt number is found in the front stagnation points of the cylinders and minimum values were found near the adiabatic boundary. Increasing either the separation distance and/or the Reynolds number leads to enhance the heat dissipation and reduce the cooling time. The separation distance parameter is mainly affecting the cooling process of the downstream cylinder which can be enhanced by increasing this distance. The results show that the cooling period to a specific temperature will takes relatively longer for the cylinders made of high thermal inertia materials like carbon steels compare to those of low thermal inertia like plywood.

\section{REFERENCES}

[1] D. Sumner, "Two circular cylinders in cross-flow: a review", Journal of Fluids and Structures, vol. 26, pp. 849-899, 2010. doi.org/10.1016/j.jfluidstructs.2010.07.001.

[2] A. Harichandan, and A. Roy, "Numerical investigation of flow past a single and tandem cylindrical bodies in the vicinity of a plane wall", Journal of Fluids and Structures, vol. 33, pp. 19-43, 2012.doi: 10.1016/j.jfluidstructs.2012.04.006.

[3] R.J. Jiang, and J.Z. Lin, "Wall effects on flows past two tandem cylinders of different diameters", Journal of Hydrodynamics, vol. 24, pp. 1-10, 2012. doi: 10.1016/s1001-6058(11)60212-6.

[4] B. Dehkordi, H. Moghaddam, and H. Jafari, "Numerical simulation of flow over two circular cylinders in tandem arrangement", Journal of Hydrodynamics, vol. 23, pp. 114-126, 2011. doi.org/10.1016/S1001-6058(10)60095-9.

[5] S. Sarkar, A. Dalal, and G. Biswas, "Mixed convective heat transfer from two identical square cylinders in cross flow at Re= 100", International Journal of Heat and Mass Transfer, vol. 53, pp. 2628-2642, 2010. doi.org/10.1016/j.ijheatmasstransfer.2010.02.053.

[6] J. Lu, H. Han, and B. Shi, "A numerical study of fluid flow passes two heated/cooled square cylinders in a tandem arrangement via lattice Boltzmann method", International Journal of Heat and Mass Transfer, vol. 55, pp. 3909-3920, 2012. doi.org/10.1016/j.ijheatmasstransfer.2012.03.010.

[7] D. Chatterjee, G. Biswas, and S. Amiroudine, "Mixed convection heat transfer from an in-line row of square cylinders in crossflow at low Reynolds number", Numerical Heat Transfer (A), vol. 61, pp. 891-911, 2012. doi: 10.1080/10407782.2012.677326.

[8] D. Chatterjee, and M. Raja, "Mixed convection heat transfer past in-line square cylinders in a vertical duct", Thermal Science, vol. 17, pp. 567-580, 2013. doi: 10.2298/TSCI101004199C.

[9] Z. Huang, G. Xi, W. Zhang, and S. Wen, "Mixed convection heat transfer from confined tandem square cylinders in a horizontal channel", International Journal of Heat and Mass Transfer, vol. 66, pp. 625-631, 2013. doi.org/10.1016/j.ijheatmasstransfer.2013.07.075.

[10] I. Harimi, and M. Saghafian, "Numerical simulation of fluid flow and forced convection heat transfer from tandem circular cylinders using overset grid method", Journal of Fluids and Structures, vol. 28, pp. 309-327, 2012. doi:10.1016/j.jfluidstructs.2011.12.006.

[11] H. Laidoudi, and M. Bouzit, "Mixed convection heat transfer from confined tandem circular cylinders in cross-flow at low Reynolds number", Mechanika, vol. 23, pp. 522-527, 2017. doi.org/10.5755/j01.mech.23.4.15258.

[12] H. Laidoudi, and O.D. Makinde, "Mixed convection heat transfer around a tandem circular cylinders in incompressible downward flow", Diffusion Foundations, vol. 16, pp. 12-20, 2018. doi:10.4028/www.scientific.net/DF.16.12.

[13] G. Juncu, "A numerical study of momentum and forced convection heat transfer around two tandem circular cylinders at low Reynolds numbers. Part I: Momentum transfer", International Journal of Heat and Mass Transfer, vol. 50, pp. 3788-3798, 2007. doi:10.1016/j.ijheatmasstransfer.2007.02.020.

[14] G. Juncu, "A numerical study of momentum and forced convection heat transfer around two tandem circular cylinders at low Reynolds numbers. Part II: Forced convection heat transfer", International Journal of Heat and Mass Transfer, vol. 50, pp. 3799-3808, 2007. doi:10.1016/j.jheatmasstransfer.2007.02.021.

[15] N. Mahir, and Z. Altaç, "Numerical investigation of convective heat transfer in unsteady flow past two cylinders in tandem arrangements", International Journal of Heat and Fluid Flow,; vol. 29, pp. 1309-1318, 2008. doi:10.1016/j.ijheatfluidflow.2008.05.001.

[16] E. Salcedo, J. Cajas, C. Trevino, and L. Suastegui, "Numerical investigation of mixed convection heat transfer from two isothermal circular cylinders in tandem arrangement: buoyancy, spacing ratio, and confinement effects", Theoretical and Computational Fluid Dynamics, vol. 31, pp. 159-187, 2017. doi: 10.1007/s00162-016-0411-z.

[17] N.H. Saeid, and B. Busahmin, "Transient cooling of a cylinder in cross flow bounded by an adiabatic wall", ASEAN Journal of Chemical Engineering, vol. 17, pp.17-26, 2017. doi: 10.22146/ajche.49552.

[18] M.A. Martins, L.S. De Oliveira, J.A.O. Saraz, "Numerical study of apple cooling in tandem arrangement", Dyna Journal, vol. 166, pp. 158-165, 2011. ISSN 0012-7353.

[19] Y.A. Cengel, and A.J. Ghajar, Heat and Mass Transfer: Fundamentals and Applications, $5^{\text {th }}$ Edition, McGraw-Hill, New York, 2015. 
[20] FLUENT User's Guide, Fluent Inc., Centerra Resource Park, 10 Cavendish Court, Lebanon, NH 03766, 2006.

[21] ANSYS FLUENT User's Guide, ANSYS Inc., Southpointe 275, Technology Drive Canonsburg,PA 15317, 2011.

[22] N.H. Saeid, "Natural convection in a square cavity with discrete heating at the bottom with different fin shapes", Heat Transfer Engineering Journal, vol. 39, pp. 154-161, 2018. doi.org/10.1080/01457632.2017.1288053.

[23] N.H. Saeid, "Numerical investigations of sand erosion in a choke valve", Journal of Mechanical Engineering and Sciences, vol. 12, pp. 3988-4000, 2018. doi.org/10.15282/jmes.12.4.2018.01.0347.

[24] N.H. Saeid, B. Busahmin, and A. Khalid, "Mixed convection jet impingement cooling of a moving plate", Journal of Mechanical Engineering and Sciences, vol. 13, pp. 5528-5541, 2019. doi.org/10.15282/jmes.13.3.2019.20.0446.

[25] J. Wang, R.L. Bras, G. Sivandran, and R.G. Knox, "A simple method for the estimation of thermal inertia", Geophysical Research Letters, vol. 37, pp. 1-5, 2010. doi.org/10.1029/2009GL041851. 